\title{
Diagnóstico a partir de la propuesta de un museo interactivo en el museo de Fray Bernardino de Sahagún
}

\author{
Diagnostic based on the the proposal of an interactive museum in the "Fray Bernardino de \\ Sahagún" museum in Tepeapulco, Hidalgo.
}

\author{
Alam Montiel García ${ }^{a}$, Mayra A. Valerio Nolasco ${ }^{b}$, Francisco O. Peña Guajardo $^{c}$
}

\begin{abstract}
:
The "Fray Bernardino de Sahagún" museum is an anthropological museum located in Tepeapulco, Hidalgo, which could be improved by transforming itself into an interactive museum to improve its services that will serve to attract tourists to the region that is full of cultural and natural wealth.

The objective was to analyze the touristic processes and the Material Cultural Heritage in the municipality to design a proposal for the creation of an interactive museum in the "Fray Bernardino de Sahagún" museum. For it, the study was carried out under a qualitative approach with phenomenological design in order to know the experiences and opinions of people.

The proposal of the interactive museum in the municipality of Tepeapulco Hidalgo is really viable, but factors such as the budget, space and public to which it is directed must be taken into account.
\end{abstract}

Keywords:

Museum, Tourism, Interactive

\section{Resumen:}

El museo de "Fray Bernardino de Sahagún es un museo antropológico ubicado en el municipio de Tepeapulco, Hidalgo el cual puede progresar al transformarse en un museo interactivo para mejorar sus servicios, que servirán para atraer turistas a la región que llenen de riqueza cultural y natural.

El objetivo fue analizar los procesos turísticos y el Patrimonio Cultural Material en el municipio para diseñar una propuesta para la creación de un museo interactivo en el museo de "Fray Bernardino de Sahagún. Para ello el estudio se realizó bajo un enfoque cualitativo con diseño fenomenológico con el fin de conocer las experiencias y opiniones de las personas.

La propuesta del museo interactivo en el municipio de Tepeapulco Hidalgo es realmente viable, pero se debe tener en cuenta factores como el presupuesto, espacio y público al que está dirigido.

\section{Palabras Clave:}

Museo, Turismo, Interactivo

\section{Introducción}

El municipio de Tepeapulco, en el estado de Hidalgo, está ubicado en los Llanos de Apan: "[...] limita al sur y oeste con el valle de México, por el norte con la Sierra de las
Navajas o Pachuca y el Valle de Tulancingo, y por el oriente con la Sierra Norte de Puebla" (Jiménez \& Ramírez, 2010, p. 188). En la zona abunda la liebre, la tuza, el conejo, el ratón de campo, la codorniz, el coyote y la víbora de cascabel entre otros, la flora está conformada

\footnotetext{
Montiel García Alam, Universidad Autónoma del Estado de Hidalgo, https://orcid.org/0000-0003-1127-232X, Email: alam.mg.2197@gmail.com

b Valerio Nolasco Mayra Anaid, Universidad Autónoma del Estado de Hidalgo, https://orcid.org/0000-0003-2155-1250, Email: mayra_valerio@uaeh.edu.mx

c Peña Guajardo Francisco Omar, Universidad Autónoma del Estado de Hidalgo, https://orcid.org/0000-0001-5582-9148, Email: francisco_pena@uaeh.edu.mx
} 
principalmente por pino y oyamel; también hay especies de encinos, huejote, madroño, matorral, sabinos, cedro blanco, tejocote entre otros. La temperatura promedio de Tepeapulco se encuentra, entre los $10.9^{\circ} \mathrm{C}$ en los meses más fríos y $16^{\circ} \mathrm{C}$ para los meses más calurosos.

Tepeapulco se caracteriza por diferentes procesos históricos culturales, cabe mencionar, que lo han marcado por tener una tradición colonial, principalmente, relacionada con el proceso de evangelización que data desde el sigloXVI. Del mismo modo, otro de los contrastes que se reproducen en este espacio geográfico, es el surgimiento de la Antropología en América, como una de las principales ciencias sociales, pioneras en el estudio de los pueblos indígenas en nuestro país, todo esto gracias al gran trabajo antropológico llamado: Historia general de las cosas de la Nueva España, escrito por Bernardo de Rivera mejor conocido como Fray Bernardino de Sahagún, y sus alumnos, trabajo el cual habla sobre la cultura y la historia de los pueblos del altiplano central de México, que se enriqueció mediante la recolección de información obtenida a través de entrevistas realizadas a personas de avanzada edad consideradas sabias en Tepeapulco, Hidalgo.

Cabe mencionar que "[...] un museo es una institución sin fines lucrativos, permanente, al servicio de la sociedad y de su desarrollo, abierta al público, que adquiere, conserva, investiga, comunica y expone el patrimonio material e inmaterial de la humanidad y su medio ambiente con fines de educación, estudio y recreo. Ante la necesidad de una institución de esta índole, en febrero de 1959 en el municipio de Tepeapulco, Hidalgo, con ayuda de la comunidad se crea el museo arqueológico "Fray Bernardino de Sahagún", el cual exhibe piezas arqueológicas de los periodos preclásico y clásico de las culturas tolteca, teotihuacana y azteca, ubicado en una parte del ex convento y templo de San Francisco de Asís (antiguo hogar de Fray Bernardino de Sahagún), construido en 1528 por los evangelizadores franciscanos" (SIC México, 2018). El museo únicamente ofrece al público servicio de visitas guiadas al lugar.

Dada la importancia histórica, tu rística y cultu ral del museo se tiene como finalidad analizar la relación entre Turismo y Patrimonio Cultural, mediante un diagnóstico de una propuesta sobre la realización de un museo interactivo en el museo de "Fray Bernardino de Sahagún".

El objetivo de evaluar la propuesta es saber, de qué manera la trasformación posibilita una mejor calidad en el servicio turístico en el municipio de Tepeapulco, Hidalgo.
Se debe tomar en cuenta que actualmente los museos están en la necesidad de renovarse museográficamente e incorporar servicios complementarios para evitar volverse obsoletos ante las necesidades contemporáneas.

La investigación se realizó a través de entrevistas semiestructuradas a personas de 8 a 65 años del municipio de Tepeapulco, Hidalgo.

\section{Unidades de investigación:}

Tepeapulco, Hidalgo:

Tepeapulco es un municipio ubicado en el Sureste del estado de Hidalgo, está ubicado en los llanos de Apan, entre los paralelos $19^{\circ} 45^{\prime} 00^{\prime \prime}$ de latitud norte y $98^{\circ} 31^{\prime} 37 \mathrm{de}$ longitud oeste del meridiano de Greenwich", al sur colinda con el valle de México, por el norte con la Sierra de las Navajas y el Valle de Tulancingo, y por el oriente con la Sierra Norte de Puebla. Cuenta con una extensión territorial de 244.07 kilómetros cuadrados (Jiménez \& Ramírez, 2010, p. 188).

El nombre de Tepeapulco proviene las raíces nahuas: tepetl, "Cerro", apul, "Agua", co "en", "Lugar rodeado de agua" O "Cerro rodeado de agua". A consecuencia de la ocupación europea en esta zona se trajo consigo la típica organización española del viejo continente como las mercedes reales, haciendas, y encomiendas, dándonos como resultado el actual paisaje hacendario que tanto caracteriza a esta región.

Existe un acueducto comúnmente llamado "Caja de agua", "[...] Se trata de uno de los ingeniosos acueductos que datan del silgo XVI. Es una construcción de planta aproximadamente cuadrada, con dos entradas enmarcadas por sendos arcos sostenidos por semicolumnas. Los dos arcos rematan en una cornisa que está labrada y donde se puede leer "Siendo bisorrey Don Antonio de Mendoza y Tlatuhuani Carlos V, siendo gobernador Don Diego de Velázquez llegó por agua la verdadera fe y el inmaculado Dios". En otra parte se leé "Aquí bisorrey Antonio de Mendoza y aquí en Tepeapulco Diego Velázquez". En los otros dos costados hay cuatro salidas de agua por muro en forma de cabezas de león, que derramaban agua en estanques rectangulares."(Cultura, 2020).

Fray Bernardino de Sahagún:

Nacido entre 1498 y 1500 en el pueblo leonés de Sahagún al norte de España, estudio en la prestigiosa universidad de Salamanca, a los 18 años se unió a la orden de San Francisco para en 1524 ordenarse sacerdote, en 1529 junto con 19 religiosos a la Nueva España, a su llegada vivió en Tlalmanalco y de ahí se movió al convento de Xochimilco y de ahí impartió clases en Tlatelolco. Entre 
1558 y 1559 en Tepeapulco, Fray Bernardino junto con sus alumnos da inicio al primer estudio antropológico formal en el nuevo mundo, el cual recibe el nombre de primeros memoriales, libro en el cual habla sobre la cultura de los habitantes del centro del país, para escribirlo recibió ayuda de locales de avanzada edad que a través de su conocimiento nutrían la gran obra de Fray Bernardino, tiempo después este libro se convertiría en su obra magna La Historia general de las cosas de Nueva España libro con el cual logra recopilar y describir todo lo que se refiere a la vida de los antiguos mexicanos (creencias religiosas, cultos, ritos, historia, calendario, fiestas, labores agrícolas, etc).

Templo y Ex convento de San Francisco:

[...] "Este edificio se encuentra localizado en la zona centro de la localidad de Tepeapulco, Hidalgo, este templo pertenece al conjunto de edificaciones mendicantes levantadas por los franciscanos en el siglo XVI. La entrada al atrio actualmente es un jardín, el conjunto está situado sobre una antigua pirámide donde aún se pueden observar algunos escalones originales mismos que conducen a una cruz atrial adosada al muro, la cruz hermana se encuentra incrustada a la mitad de la parte superior del muro izquierdo de la nave principal, ambas cruces están realizadas en piedra gris labrada, el recinto tiene una altura que sobrepasa los siete metros sobre el nivel de la calle. La fachada del edificio está compuesta por un arco de menos de medio punto soportado por dobles pilastras de estilo gótico, el claustro alto y bajo tiene un contorno cuadrado, el primer piso cuenta con cinco arcadas de medio punto, en cada lado, su cubierta es de viguería de cedro. La pintura mu ral que se encuentra por casi todo el edificio es de gran valor cultural ya que en las obras plasmadas se puede observar arte tequitqui símbolo de la opresión y mezcla de dos culturas abismalmente diferentes. En una de las celdas vivió Bernardino de Sahagún quien entre 1558 y 1560 realizo en la localidad de Tepeapulco el primer estudio antropológico formal en el nuevo mundo, información que recibió el nombre de primero memoriales, mismos que culminarían con la gran obra conocida como La Historia General de las Cosas de la Nueva España." (INAH, 2019).

Museo de Fray Bernardino de Sahagún:

[...] "Fundado por la comunidad de Tepeapulco en 1959 con el objetivo de salvaguardar y mostrar su legado histórico, el museo es nombrado Fray Bernardino de Sahagún en honoral sacerdote y escritor con el mismo nombre. El museo se encuentra en el claustro bajo del ex convento, en el antiguo comedor de los sacerdotes, el museo cuenta con cuatro salas de exhibición, la primera sala está destinada a exposiciones temporales, la segunda sala habla sobre el área geo cultural denominada Mesoamérica,

La tercera sala muestra una excavación arqueológica donde se puede observar parte del sistema hidráulico del ex convento construido en el siglo XVI, en los muros se exhibe parte del acervo virreinal del convento con importantes pinturas al óleo como la de San Francisco de Asís, La Buena Muerte de San Agustín, entre otras, la tercera sala está dedicada al Horizonte Posclásico la cual contiene elementos en cerámica de la cultura mexica y material lítico como un disco solar donde aparece representado Tonatiuh, deidad solar y en la cuarta sala se exhibe la importancia que tuvo la religión en la vida del pueblo mexica, ya que se pueden encontrar esculturas como la de Tláloc (Dios de la lluvia), Chicomecóatl (deidad del maíz) y Xiuhtecutli (dios del año), al final de la sala se encuentra la pieza estrella del museo, que es un monolito de Nahui Ollín, símbolo abstracto del calendario mexica". (INAH, 2019).

\section{ICOM:}

La ICOM es una organización internacional de museos y profesionales de los museos cuya finalidad es in vestigar, perpetuar, perennizar y transmitir a la sociedad el patrimonio cultural y natural mundial, presente y futuro, tangible e intangible.

\section{Museo:}

ICOM tiene la siguiente definición: [...] "Ios museos son espacios democratizadores, inclusivos y polifónicos para el diálogo crítico sobre los pasados y los futuros. Reconociendo y abordando los conflictos y desafíos del presente, custodian artefactos y especímenes para la sociedad, salvaguardan memorias diversas para las generaciones futuras, y garantizan la igualdad de derechos y la igualdad de acceso al patrimonio para todos los pueblos. Los museos no tienen ánimo de lucro. Son participativos y transparentes, y trabajan en colaboración activa con y para diversas comunidades a fin de coleccionar, preservar, investigar, interpretar, exponer, y ampliar las comprensiones del mundo, con el propósito de contribuir a la dignidad humana y a la justicia social, a la igualdad mundial y al bienestar planetario."(ICOM, 2019).

\section{El museo comunitario:}

El museo sirve como herramienta para proteger el patrimonio cultural de una comunidad, en el caso de un museo comunitario sirve para proteger y mostrar a través de exhibiciones el patrimonio cultural del lugar, estos suelen crearse a través de contribuciones de los pobladores de una comunidad. El museo comunitario genera acciones en favor de los intereses de la población, fortalece la capacidad de organización local en redes, 
brinda capacitación y responde a necesidades comunitarias. [...] "En México, el origen de este tipo de proyecto de museo comunitario, detalló la antropóloga Teresa Morales, se remonta a 1985 en Santa Ana del Valle, comunidad zapoteca localizada en el Valle de Tlacolula de Matamoros, Oaxaca, que demandaba apoyo para crear un recinto que resguardara su patrimonio cultural (INAH, 2015).

En 1986, como fruto del trabajo y organización de la población de Santa Ana, se creó el museo Shan-Dany, que en zapoteco significa "bajo el cerro", conformado con el apoyo y la asesoría del INAH Oaxaca. Atesora más de un centenar de objetos arqueológicos e históricos, entre los que destacan los restos de un entierro prehispánico, armamento utilizado durante la Revolución Mexicana, un antiguo telar de madera y fotografías.

En México existen alrededor de medio centenar de museos comunitarios en estados como Oaxaca, Yucatán, Veracruz, Morelos, Tlaxcala, Hidalgo, Guerrero, Querétaro y Puebla, que nacieron a partir del interés de las poblaciones por preservar su patrimonio, memoria e identidad, señalaron los antropólogos Cuauhtémoc Camarena y Teresa Morales, en el Seminario Permanente de Museología en América Latina. Museo(s) y Comunidad(es)" (INAH, 2015).

\section{El museo interactivo:}

Todo aquello que desee sobrevivir el paso del tiempo necesita evolucionar constantemente y el museo no es la excepción, por ende el papel y esencia del museo ha cambiado drásticamente a través del tiempo, un aspecto importante a tratar son las ventajas que implica un museo interactivo, puesto que en él se pondera la educación con el juego, esto resulta muy beneficioso ya que los usuarios del museo aprenden de una forma vivencial provocando que ellos absorban de mejor manera el conocimiento adquirido, esto a causa de la implicación de sentidos como el tacto, la vista y el oído.

En un museo interactivo no se invita al público a copiar o a imitar, lo que busca es fortalecer la curiosidad, mientras se refuerzan las capacidades de análisis, reflexión, discusión, expresión y gestión de los participantes en el proceso educativo dentro de los museos.

\section{Población y muestra:}

La población de estudio estuvo representada por 68 personas de 8 a 65 años del municipio de Tepeapulco, Hidalgo, tomando en cuenta la población del municipio que es de 49850 para saber la cantidad de personas a encuestar se utilizó la siguiente formula, teniendo en cuenta un margen de error del $10 \%$.

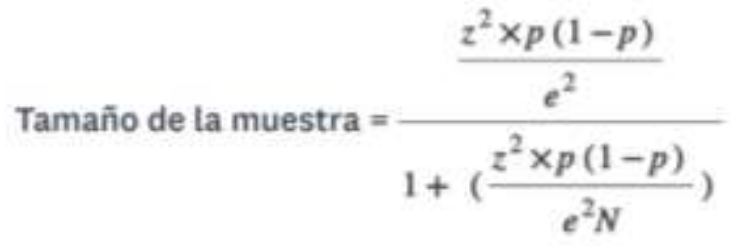

$\mathrm{N}$ = tamaño de la población

$\mathrm{e}=$ margen de error (porcentaje expresado con decimales)

$z=$ Nivel de confianza $(90 \%=1.65)$

$\mathrm{p}=$ Prevalencia esperada del parámetro a evaluar, en caso de desconocerse $(p=0.5)$,

Con esta muestra se buscó mostrar distintas perspectivas del fenómeno estudiado ya que su complejidad lo ameritaba.

\section{Resultados}

Los resultados se obtuvieron a través de encuestas realizadas en el municipio de Tepeapulco, Hidalgo, los resultados mostraron que las personas están interesadas en las cualidades que los museos interactivos ofrecen, puesto que la mayoría concluyo que la narrativa e interacción que los guías del museo proporcionaban hacían realmente interesante la visita al museo.

Mostraron un interés común en los talleres, comentaron que les causa atracción este tipo de actividades poco usuales.

El museo de Fray Bernardino de Sahagún tiene una buena imagen entre los visitantes.

Como se puede observar en la gráfica 1 , el $60 \%$ de los visitantes del museo coinciden en que el museo es bueno, el $30 \%$ cree que es excelente, el $7 \%$ tuvo una opinión neutral al considerarlo ni bueno ni malo y solamente el $1 \%$ piensa que es malo

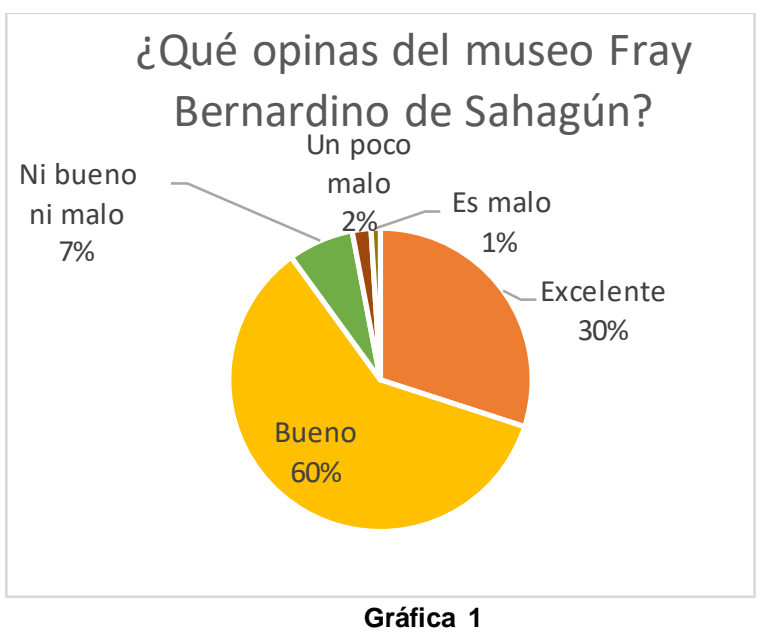


La gráfica 2 muestra que los principales motivos de las visitas al museo de Fray Bernardino de Sahagún son en primer lugar el lúdico con un $39 \%$, en segundo lugar, se encuentra la curiosidad con un $34 \%$ representada principalmente por niños de una edad de 7 a 10 años, quienes por mera curiosidad ingresan al museo sin saber nada del contexto o temática del museo y en tercer lugar, se encuentra el escolar con un $27 \%$.

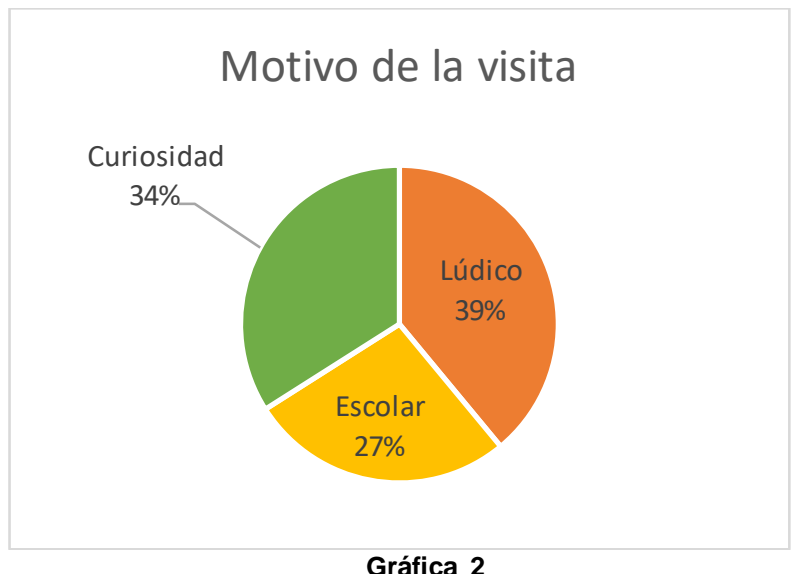

Gráfica 2

La gráfica 3 nos muestra que solamente el $32 \%$ de las personas encuestadas afirmaron conocer que es un museo interactivo e incluso en algunos casos haber asistido a alguno, el 68\% restante negó conocer alguna característica de este tipo de museos.

\section{¿Sabes que es un museo interactivo?}

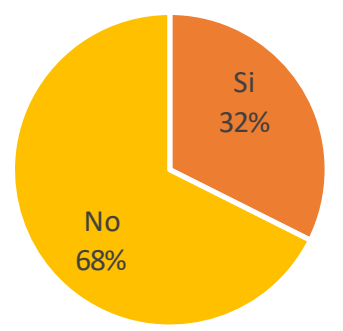

Gráfica 3

La gráfica 4 señala que la actividad que más gustó entre los entrevistados fue la excavación arqueológica con un $34 \%$, el taller de preparación con un $26 \%$ agradó mucho a los niños y personas de la tercera edad, el taller de preparación de chocolate con $26 \%$ fue la preferida por las personas de entre 13 y 25 años, al final con un $15 \%$ se encuentra el taller de escultura en barro

\section{¿Qué actividad te atrae más?}

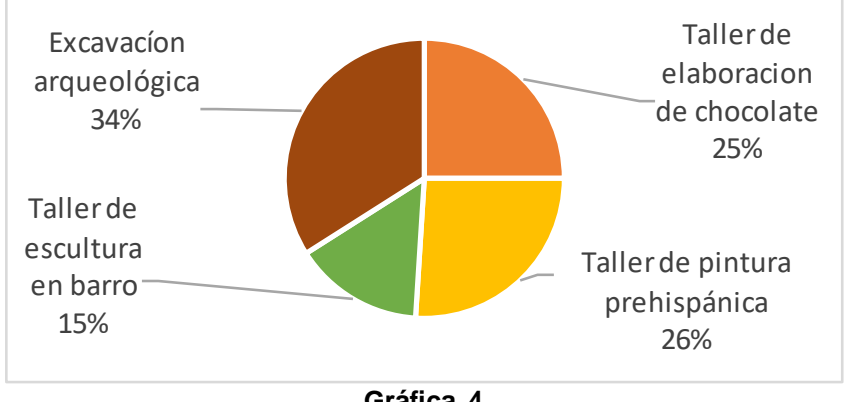

Gráfica 4

Los niños de 8 a 13 años se mostraron realmente interesados en el museo el cual dicen disfrutar pero no comprender del todo ya que las tarjetas informativas que se encuentran en el museo no son leídas por ellos, pero las piezas arqueológicas les generan interés, el conocimiento que han adquirido ha sido principalmente por las narrativas de los guías y explicaciones vagas de los padres de familia. Los niños al igual que los padres de familia se mostraron entusiasmados ante la posibilidad de tener actividades en el museo, principalmente en la de escarbar piezas arqueológicas, los padres de familia visualizaron la propuesta del museo interactivo como un sitio lúdico en el cual sus hijos se llenarían de cultura.

Las personas de 13 a 20 años no estaban interesadas en el museo ya que lo consideraban aburrido y silencioso, sus visitas al museo han sido resultado de tareas escolares. Mostraron especial interés en la propuesta de un museo interactivo puesto que la posibilidad de tener elementos audiovisuales, participar en un juego de pelota y tener talleres en el museo haría más divertida e interesante la visita.

Las personas de 22 a 40 años consideran muy bueno el museo, suelen leer las láminas informativas pero su visita es rápida y con poca profundidad. Este segmento se mostró relativamente interesado puesto que tienen la creencia de que los museos interactivos están enfocados principalmente a niños, pero se mostraron intrigados ante la posibilidad de elementos audio visuales y de talleres gastronómicos.

Las personas de 40 a 65 años son las que más tiempo pasan en el museo y a menudo solicitan una visita gu iada, es común que lo hagan en grupos y con anticipación, usualmente lo hacen en tours turísticos con el objetivo principal de visitar el ex convento donde se encuentra el museo. Mostraron especial interés ante la posibilidad de 
talleres de artesanías y pintura ya que son actividades que disfrutan hacer.

Los guías del museo comentaban que no se necesita mucho dinero para tener actividades propias de un museo interactivo, también comentaron que el espacio en el mismo dificulta algunas actividades que se podrían llegar a hacer pero que no lo hacía imposible, además las regulaciones y el recorte presupuestal al INAH realizado en el presente sexenio representaba un gran reto, afortunadamente a pesar de los recortes presupuestales el museo obtuvo recursos para restauración que pocos sitios de la misma índole pueden tener. Los guías del museo expresaron su interés en la propuesta enriqueciendo enormemente con una propuesta de un arenero que fungiría como zona de excavación en una ampliación que el museo estaría por tener.

\section{Conclusiones}

El museo de Fray Bernardino de Sahagún cuenta con un gran potencial turístico gracias a la riqueza cultural e histórica donde está construido. El museo usualmente recibe una baja cantidad de visitantes (un promedio de 30 personas al día). Actualmente se necesita tomar acciones que ayuden a incrementar el flujo de visitantes al museo, el museo interactivo es una excelente acción que puede ayudar a aumentar el flujo.

La propuesta del museo interactivo en el museo de "Fray Bernardino de Sahagún" con base en la literatura, encuestas y entrevistas realizadas lo hacen viable, pero se debe tener especial cuidado en el tipo de actividades a realizarya que hay factores como el presupuesto, espacio y público al que va dirigido que lo limitan,

Los elementos audiovisuales fueron de especial interés para la mayor parte del público siendo una actividad potencialmente aceptable y viable, junto con la zona de excavación que atraería al público infantil y al mismo tiempo al público adulto, estos elementos deben ser tomados en cuenta si se desea modificar el museo.

\section{Referencias}

[1] Damián, M. A. (2006). Tepeapulco Región en perspectiva. Pachuca: Académica.

[2] Bourdieu, P. (1990). La distinción. Taurus. CDMX. (2016). Recuperado de https://www.turismo.cdmx.gob.mx/storage/app/media/Estadisticas/Perf il $\% 20$ del $\% 20$ Turista \%202016/EST\%20IPNSECTUR\%20CDMX\%20 Perfil\%202016\%20Reporte\%20Ejecutivo.pdf.

[3]DATATUR. (2019). Recuperado de https://www.datatur.sectur.gob.mx/SitePages/ActividadesCulturales.as $\mathrm{px}$
[4] INAH. (2019). Recuperado de https://www.estadisticas.inah.gob.mx/.

[5] Megale, A. A. (2001). REDALYC. Recuperado de https://www.redalyc.org/pdf/844/84420403.pdf.

[6] Hernández, S. (s.d.). UNESCO. Recuperado der http://www.lacult.unesco.org/docc/evolucion_museos.pdf.

[7] ICOM. (2007). Recuperado de https://icom.museum/es/actividades/normas-.

[8] Orozco, G. (2015). REDALYC. Recuperado de http://www.redalyc.org/html/998/99815914005/index.html

[9] UNESCO. (s.d.). Recuperado de https://es.unesco.org/creativity/sites/creativity/files/digital-.

[10] SIC. México. (s.d.). Recuperado de https://sic.cultura.gob.mx/lista.php?table=museo\&estado_id=13\&muni cipio_id=-1.

[11] Hernández Sampieri, R. (2010). Metodología de la investigación. Ciudad de México: MCGRAW-HIL.

[12] Cultura, S, d. (20 de ABRIL de 2020). CULTURA. Obtenido de https://pa trimonioculturalyturismo.cultura.gob.mx/destino_mes/tepeapulco /otros.html

[13] ICOM. (s.f.). ICOM. Obtenido de https://icom.museum/es/news/elicom-anuncia-la-definicion-alternativa-del-museo-que-se-sometera-avotacion/

[14] INAH. (23 de OCTUBRE de 2015). NAH. Obtenido de https://www.inah.gob.mx/en/boletines/4434-museos-comunitariospreservan-la-memoria-e-identidad

[15] INAH. (14 de FEBRERO de 2019). INAH. Obtenido de https://www.inah.gob.mx/red-de-museos/274-ex-convento-de-tepeapulco 